\title{
Pichia fabianii blood infection in a premature infant in China: case report
}

Yuan $\mathrm{Wu}^{1 \dagger}$, Jing Wang ${ }^{2 \dagger}$, Wenge $\mathrm{Li}^{1}$, Hongbin $\mathrm{Jia}^{2}$, Jie Che ${ }^{1}$, Jinxing $\mathrm{Lu}^{1}$, Lanzheng Liu ${ }^{3^{*}}$ and Ying Cheng ${ }^{{ }^{*}}$

\begin{abstract}
Background: Invasive fungal infections caused by uncommon fungi have increased in recent years. Hospitalized low-birth-weight infants are at high risk for neonatal fungal infections. Pichia fabianii is a rare pathogen causing blood infection, which has reportedly caused only 4 cases of fungemia and 1 case of endocarditis worldwide. Here, we describe the first case of a P. fabianii blood infection in a premature infant in China.

Case presentation: On July $28^{\text {th }}$, a low-birth-weight (LBW, $1760 \mathrm{~g}$ ) female infant born at $33^{+4}$ weeks of gestation was admitted to the pediatric intensive care unit with mild neonatal asphyxia. Until August $2^{\text {nd }}$, a mechanical respirator was used to assist respiration under the Continuous Positive Airway Pressure (CPAP) model. The baby had an increased body temperature and a fever. To prevent infection, Ceftriaxone Sodium (CS) was administered intravenously for three days, after which Cefepime was administered until August $13^{\text {th }}$. Chest X-rays showed suspected plaque-like shadows in the right lung. Blood cultures twice tested positive for fungal infection caused by Candida pelliculosa (recognized as Pichia fabianii later), which is first mis-identified by commercial kit. Hence, intravenous fluconazole was administered. However, cultures of other body fluids (e.g., urine, feces and sputum) tested negative for fungal infection. Routine tests and biochemistry of cerebrospinal fluid (CSF) were normal. Latex agglutination of Cryptococcus neoformans and fungi cultures in the CSF were also negative. After 14 days of intravenous fluconazole, blood was re-cultured, the result of which was negative. On August $30^{\text {th }}$, intravenous fluconazole was suspended. On Sep $3^{\text {rd }}$, the infant left the hospital in good health.

Conclusions: This is the first case of a blood infection caused by P. fabianii in a LBW premature female infant in China. Risk factors for fungal infection include premature birth, as well as mechanical invasive operation and antibacterial drug usage. Whether such risk factors necessitate prophylactic use of antifungal drugs is an important question that has yet to be fully addressed. Additionally, the pathogen P. fabianii collected in this study was resistant to amphotericin $B$ (AMB) and itraconazole (ITR). With the exception of the azole-resistant endocarditis case, all other cases have not demonstrated such a resistance. Finally, commercial biochemical methods used in routine practice are limited in their ability to identify P. fabianii. Molecular genetic based methods are imperative for identification of uncommon fungal species from disseminated infections.
\end{abstract}

Keywords: Pichia fabianii, Fungemia, Infant infection

\footnotetext{
* Correspondence: jinanliu2005@126.com; chengying@icdc.cn

${ }^{\dagger}$ Equal contributors

${ }^{3}$ Municipal Centre for Disease Control and Prevention, Jinan, Shandong,

China

'Department of Hospital Acquired Infection Control and Prevention, National, Institute for Communicable Disease Control and Prevention, Chinese Center for Disease Control and Prevention, State Key Laboratory for Infectious Disease Prevention and Control, Beijing, China

Full list of author information is available at the end of the article
} 


\section{Background}

Fungal infections are an increasing threat for: hospitalized patients using catheters (especially vascular), the immunocompromised (e.g., patients with cancer or HIV), premature neonates, and the elderly $[1,2]$. Low birth weight (LBW, $<2500 \mathrm{~g}$ ) is particularly neonates have an increased risk of fungal infection. Reports of invasive infections caused by uncommon fungi have increased in recent years [3,4]. Pichia, teleomorph stages of several Candida species, is an ascomycetous yeast species rarely involved in human infections [5]. Here we describe the first case of a fungemia caused by Pichia fabianii (also termed as Candida fabianii or Lindnera fabianii) in a premature female neonate in Beijing, China.

\section{Case presentation}

A female infant, born at $33^{+4}$ weeks of gestation, was admitted to the pediatric intensive care unit at a general hospital in Beijing on July 28, 2010. She was diagnosed as premature and LBW (1760 g), with mild neonatal asphyxia. The mother was an elderly primipara (33years-old), with a hysteromyoma, who had developed gestational hypertension. The mother had no history of infectious or other underlying disease. Due to low implantation of the placenta in the mother's uterus, the baby had been delivered via caesarean. The infant was kept in a premature incubator $\left(33-35^{\circ} \mathrm{C}\right)$, and was given multi-function intensive care, as well as prohibition of food and drink for $20 \mathrm{~h}$, proper intravenous fluids according to need, and peripheral venous hyperalimentation. A respirator was used to assist breathing until August $2^{\text {nd }}$. To prevent infection, CS was administered intravenously (70 mg Qd) for three days, at which point cefepime was used instead. On August $5^{\text {th }}$, the incubator was aborted and inspired oxygen was given. On August $11^{\text {th }}$, the infant had a fever of $37.7^{\circ} \mathrm{C}$, cold hands and feet, and a heart rate of 160-180 bpm; blood cultures were tested and cefepime was added to $140 \mathrm{mg}$ Qd. On August $12^{\text {th }}$, chest X-Rays revealed plaque-like shadows in the right lung. Blood, urine, feces and sputum specimens were collected and sent for culturing and further identification. Blood cultures indicated fungal infection (Candida pelliculosa), though all other bodily fluids tested negative in this respect. On August $13^{\text {th }}$, intravenous fluconazole (10 mg per day, micro pump $5 \mathrm{ml} / \mathrm{h}$ ) treatment was begun and Cefepime treatment was aborted. The following day, the baby's body temperature returned to normal and her heart rate was $148 \mathrm{bpm}$. The urine smear test was still negative, and blood was collected for re-examination. After treatment with intravenous fluconazole for 10 days, blood was retested. A lumbar puncture was performed to get the CSF, the routine test (color, clarity, TP, Glu and CI) and biochemistry of which was normal. A latex agglutination test of
Cryptococcus neoformans and a fungi culture of the CSF were both negative. No abnormal symptoms were found by abdominal examination. On August $26^{\text {th }}$, blood cultures came back negative. On August $30^{\text {th }}, 18$ days after it had been begun, intravenous fluconazole administration was stopped. On September $3^{\text {rd }}$, the infant was released from the hospital in good health.

A drug sensitivity test was performed using ATB Fungus3 (BioMerieux, France). The MIC of this pathogen to 5 systemic antifungals was as follows: 5 -flucytosine, $5 \mathrm{FC}$ $(<4)$, amphotericin B, AMB $(=1)$, fluconazole, FCA $(\leq 1)$, itraconazole, ITR $(=2)$ and voriconazole, VRC $(=0.125)$, according to the manufacture's procedure. The strain was sensitive to 5FC, FCA and VRC, but resistant to ITR, S/R to AMB following the interpretation of the susceptibility test. The yeast was sent to the laboratory in Chinese Center for Disease Control and Prevention (China CDC), and was cultured on Sabouraud's Dextrose Agar, SDA (OXOID, UK) at $25^{\circ} \mathrm{C}$ for $48 \mathrm{~h}$. A single white colony was picked and subcultured on a CHROMagarCandida plate (CHROMagar, France) at $30^{\circ} \mathrm{C}$ for $48 \mathrm{~h}$, at which point the pink-white colonies were visible. This organism was capable of growing at $37^{\circ} \mathrm{C}$ and $42^{\circ} \mathrm{C}$. Carbohydrate fermentations and assimilations of the pathogen, performed using API 20C AUX (BioMerieux, France), indicated high scores for $C$. pelliculosa infection. Germ tube tests of this organism, by inoculating several colonies into a bovine serum and incubating the suspension at $37^{\circ} \mathrm{C}$ for $2 \mathrm{~h}$, were negative. After inoculation on meal agar with Tween 80 at $28^{\circ} \mathrm{C}$ for $24 \mathrm{~h}$, pseudohyphae and budding yeast could be seen. The 26S ribosomal DNA and internal transcribed spacer (ITS) of this organism was amplified, sequenced and blasted, showing high similarity to P. fabianii. The universal primer pairs ITS1 (TCCGTAGGTGAACCT GCG G) and ITS4 (TCCTCCGCTTATTGATATGC), NL (GC ATATCAATAAGCGGAGGAAAAG) and NL-4 (GGTC CGTGTTTCAAGACGG) were used to amplify these two DNA fragments [6]. The GenBank accession numbers of ITS and 26S were JQ342083 and JQ342084, respectively. MICs for AMB and ITR were re-determined by micro-dilution methods, as described by the Clinical and Laboratory Standards Institute (CLSI) [7] of China's CDC. Results showed that this pathogen was AMB and ITR resistant, coinciding with results from the commercial kit.

\section{Conclusions}

In this report, we are the first to describe the isolation of P. fabianii from the blood culture of a LBW infant in a Neonate Intensive Care Unit (NICU) in China. To our knowledge, only two cases of fungemia caused by $P$. fabianii in a preterm neonate have been reported worldwide (one in the USA [3] and one in France [5]). In the 
first case, the 5-week-old female neonate was born at $25^{+3}$ weeks of gestation to a healthy, 33-year-old mother [3]. This baby was given multiple courses of antibacterial antibiotics, mechanical ventilation and central catheter nutrition for her severe constitutional symptoms [3]. In the second case, the female infant was born at 24 weeks of gestation with an extremely low birth weight (ELBW, $<1000$ g) [5]. Central venous catheters were placed and cefotaxime and amikacin were used intravenously for a week in order to remove bacteria from bodily fluids [5]. In our case, the patient was also a premature baby girl, born at $33^{+4}$ weeks of gestation, with a LBW $(1760 \mathrm{~g})$. A respirator was applied to treat the neonatal asphyxia and antibacterial drugs were given for elevated body temperature and presumed infection. All three cases of neonate fungemia caused by $P$. fabianii occurred in a premature female infant, who had experienced invasive operation, and was treated with antibacterial drugs; all of these common characteristics are incidentally risk factors for fungal infection. This begs the questions: Is antifungal therapy necessary when these risk factors exist? In one case, mechanical operations were terminated when the fungal infection appeared and $\mathrm{AMB}$ was used successfully. Whether these behaviors are more important for the recovery of the patient is unknown [3].

Three other human infection cases have been reported $[1,2,4]$, all of which were in adults: a 40-year-old male, a 46-year-old male, and a 53-year-old female. P. fabianii caused endocarditis in the first patient and fungemia in the other two. In these previously reported 5 cases $(2$ children, 3 adult), the organism was susceptible to AMB, 5FC and FCA, except for in the endocarditis case, in which the pathogen developed in vitro resistance to azoles in the course of antifungal treatment. In our case, $P$. fabianii, isolated from the blood before an antifungal was used, showed resistance to ITR and AMB. The molecular explanation for this strain's different antifungal susceptibility necessitates further study.

Our case further allows us to study P. fabianii and its ability to cause invasive infection. NICU infants are a population at risk for developing invasive candidiasis [8]. Since $P$. fabianii has not been included in widely used commercial yeast diagnostic kits (API 20C AUX, ID32C and Vitek-2), it is difficult for clinicians to properly identify and thus has been commonly misidentified as $P$. anomala or C. utilis [9] only using commercial kit. In this study, the pathogen was initially identified as C. pelliculosa by API 20C AUX kit. And according to the molecular method mentioned in previous reports [1-4], the sequences of ITS region and D1/D2 variable region of the $26 \mathrm{~S}$ rDNA were sequenced and BLAST in GenBank. The pathogen was subsequently identified as P. fabianii.

In summary, we report the first case of blood infection caused by P. fabianii of a LBW premature female infant in China. Because preterm birth, mechanical invasive operation, and usage of antibacterial drugs are risk factors for fungal infection, the prophylactic use of antifungal drugs should be considered under such risk factors. The pathogen P. fabianii collected in this study was resistant to AMB and ITR; this resistance was not present in any other cases of $P$. fabianii except the endocarditis case, in which the pathogen was resistant to azoles [3]. Molecular genetic based methods are imperative for future identification of uncommon fungal species from disseminated infections.

\section{Consent}

Written informed consent was obtained from the patient's guardian for publication of this case report and any accompanying images. A copy of the written consent is available for review by the Editor-in-Chief of this journal.

\section{Competing interests}

The authors declare that they have no competing interests.

\section{Authors' contributions}

YW performed and interpreted all mentioned culture methods and molecular identification methods of the pathogen, and wrote the manuscript. JW and HBJ did the clinical identification of the pathogen, and collect the materials of the patient. WGL and JC took part in the culture of the pathogen. $L Z L$ and $Y C$ revised the manuscript. All authors read and approved the final manuscript.

\section{Acknowledgements}

This work was supported under the Project Sponsored by the Young Scholar Scientific Research Foundation of China CDC (2012A107) and National SciTech Key Project (2012ZX10004-201).

\section{Author details}

'Department of Hospital Acquired Infection Control and Prevention, National, Institute for Communicable Disease Control and Prevention, Chinese Center for Disease Control and Prevention, State Key Laboratory for Infectious Disease Prevention and Control, Beijing, China. ${ }^{2}$ China-Japan Friendship Hospital, Beijing, China. ${ }^{3}$ Municipal Centre for Disease Control and Prevention, Jinan, Shandong, China.

Received: 4 December 2012 Accepted: 27 February 2013 Published: 4 March 2013

\section{References}

1. Valenza G, Valenza R, Brederlau J, Frosch M, Kurzai O: Identification of Candida fabianii as a cause of lethal septicaemia. Mycoses 2006, 49:331-334.

2. Hamal P, Ostransky J, Dendis M, Horváth R, Ruzicka F, Buchta V, Vejsova M, Sauer $P$, Hejnar $P$, Raclavsky $V$ : A case of endocarditis caused by the yeast Pichia fabianii with biofilm production and developed in vitro resistance to azoles in the course of antifungal treatment. Med Mycol 2008, 46:601-605.

3. Bhally HS, Jain S, Shields C, Halsey N, Cristofalo E, Merz WG: Infection in a neonate caused by Pichia fabianii: importance of molecular identification. Med Mycol 2006, 44:185-187.

4. Gabriel F, Noel T, Accoceberry l: Lindnera (Pichia) fabianii blood infection after mesenteric ischemia. Med Mycol 2012, 50:310-314.

5. Grenouillet F, Millon L, Chamouine A, Thiriez G, Schulze O, Leroy J: Pichia fabianii Fungemia in a neonate. Pediatr Infect Dis J 2010, 29:191.

6. White T, Bruns T, Lee S, Taylor J: Amplification and direct sequencing of fungal ribosomal RNA genes for phylogenetics. PCR Protocols: A Guide to Methods and Applications. San Diego, CA: Academic Press; 1990:315-322. 
7. Pfaller MA, Rhine-Chalberg J, Barry AL, Rex JH: Strain variation and antifungal susceptibility among bloodstream isolates of Candida species from 21 different medical institutions. Clin Infect Dis 1995, 21:1507-1509.

8. Hudome SM, Fisher MC: Nosocomial infections in the neonatal intensive care unit. Curr Opin Infect Dis 2001, 14:303-307.

9. Thuler LC, Faivichenco S, Velasco E, Martins CA, Nascimento CR, Castilho IA: Fungaemia caused by Hansenula anomala an outbreak in a cancer hospital. Mycoses 1997, 40:193-196

doi:10.1186/1756-0500-6-77

Cite this article as: Wu et al:: Pichia fabianii blood infection in a

premature infant in China: case report. BMC Research Notes 2013 6:77.

\section{Submit your next manuscript to BioMed Central and take full advantage of:}

- Convenient online submission

- Thorough peer review

- No space constraints or color figure charges

- Immediate publication on acceptance

- Inclusion in PubMed, CAS, Scopus and Google Scholar

- Research which is freely available for redistribution 